PDFlib PLOP: PDF Linearization, Optimization, Protection

Page inserted by evaluation version www.pdflib.com - sales@pdflib.com 


\title{
Oxidative Stress and Programmed Cell Death in Diabetic Neuropathy
}

\author{
ANDREA M. VINCENT, ${ }^{a}$ MICHAEL BROWNLEE, ${ }^{b}$ AND JAMES W. RUSSELL ${ }^{a}$ \\ ${ }^{a}$ Department of Neurology, University of Michigan, Ann Arbor, Michigan 48109, USA \\ ${ }^{b}$ Diabetes Research Center, Albert Einstein College of Medicine, \\ Bronx, New York 10461, USA
}

\begin{abstract}
Recent evidence in both animal models and human sural nerve biopsies indicates an association with oxidative stress, mitochondrial (Mt) membrane depolarization (MMD), and induction of programmed cell death (PCD). In streptozotocin (STZ)-treated diabetic rats, hyperglycemia induces typical apoptotic changes as well as swelling and disruption of the Mt cristae in diabetic dorsal root ganglion neurons (DRG) and Schwann cells (SC), but these changes are only rarely observed in control neurons. In human sural nerve biopsies, from patients with diabetic sensory neuropathy, there is transmission electromicrograph evidence of swelling and disruption of the Mt and cristae compared to patients without peripheral neuropathy. In human SHSY5Y neurons, rat sensory neurons, and $\mathrm{SC}$, in vivo, there is an increase in reactive oxygen species (ROS) after exposure to $20 \mathrm{mM}$ added glucose. In parallel, there is an initial Mt membrane hyperpolarization followed by depolarization (MMD). In turn, MMD is coupled with cleavage of caspases. Various strategies aimed at inhibiting the oxidative burst, or stabilizing the $\Delta \Psi_{M}$, block induction of PCD. First, growth factors such as NGF can block induction of ROS and/or stabilize the $\Delta \Psi_{\mathrm{M}}$. This, in turn, is associated with inhibition of PCD. Second, reduction of ROS generation in neuronal Mt prevents neuronal PCD. Third, up-regulation of uncoupling proteins (UCPs), which stabilize the $\Delta \Psi_{M}$, blocks induction of caspase cleavage. Collectively, these findings indicate that hyperglycemic conditions observed in diabetes mellitus are associated with oxidative stress-induced neuronal and SC death, and targeted therapies aimed at regulating ROS may prove effective in therapy of diabetic neuropathy.
\end{abstract}

KEYWORDS: oxidative stress; diabetes; apoptosis; mitochondria; neuropathy

\section{INTRODUCTION}

Increasing evidence indicates that mitochondria $(\mathrm{Mt})$ are intimately associated with initiation of programmed cell death (PCD) in neurons (see Ref. 1 for review) and that Mt membrane depolarization (MMD) is associated with induction of reactive oxygen species (ROS) and initiation of PCD. Cells use free radical scavengers

Address for correspondence: James Russell, M.D., M.S., Department of Neurology, University of Michigan, 200 Zina Pitcher Place, 4414 Kresge III, Ann Arbor, MI 48109-0588. Voice: 734-763-7276; fax: 734-763-7275.

jruss@umich.edu

Ann. N.Y. Acad. Sci. 959: 368-383 (2002). ( 2002 New York Academy of Sciences. 
to detoxify superoxide and hydroxyl radicals and thereby prevent cellular injury due to oxidative stress. Detoxification of $\mathrm{H}_{2} \mathrm{O}_{2}$ occurs primarily in the $\mathrm{Mt}$, where it is reduced by glutathione. Reduction of $\mathrm{H}_{2} \mathrm{O}_{2}$ generates an oxidized glutathione disulfide that, in turn, must be reduced by NADPH to regenerate glutathione. With hyperglycemia, glucose flux generates sorbitol, which is converted to fructose and depletes the NADPH needed for regenerating glutathione, thus resulting in Mt dysfunction. ${ }^{2}$ Increased metabolic flux in the Mt due to high glucose results in increased formation of ROS such as superoxides, peroxinitrites, and highly reactive hydroxyl radicals. ${ }^{3}$ These ROS are associated with membrane lipid peroxidation, nitration of proteins (including tyrosine residues), and degradation of DNA, all of which are associated with induction of apoptosis. ${ }^{4}$ In neurons, accumulation of NADH coupled with failure of the Mt creatine phosphate pump to regenerate ATP from ADP also results in disruption of the Mt electron transfer chain and depletion of ATP associated with apoptosis. ${ }^{1}$ Although ROS has been associated with animal models of diabetes, it is unclear if oxidative stress is also important in human diabetic neuropathy. Further, the mechanisms linking oxidative stress to PCD and ultimately dorsal root ganglion (DRG) neuronal and Schwann cell (SC) loss in diabetic neuropathy are essentially unknown and will be discussed below.

\section{METHODS}

Dissociated embryonic DRGs from Harlan Sprague-Dawley rats were aseptically plated on air-dried, collagen-coated ACLAR dishes in the presence of serum- and insulin-free media and $10 \mathrm{ng} / \mathrm{mL}$ nerve growth factor (NGF) as previously described. ${ }^{5,6}$ SH-SY5Y human neuroblastoma cells were grown in DMEM with $10 \%$ calf serum at $37^{\circ} \mathrm{C}$ in a humidified atmosphere containing $10 \% \mathrm{CO}_{2}$ as previously described. ${ }^{7}$ In experiments, cells were subcultured in DMEM alone with the experimental condition. SC were cultured as previously described. ${ }^{8}$ SH-SY5Y neurons and SC do not normally require NGF for survival.

To obtain tissue samples, animals were perfused prior to death with Trump's fixative (4\% paraformaldehyde and $1 \%$ glutaraldehyde in $0.1 \mathrm{M}$ phosphate buffer) by intracardiac infusion. ${ }^{6}$ The whole vertebral column was removed and a 4- to 5$\mathrm{mm}$ strip of bone was excised from the dorsal roof of the vertebral column as previously described. ${ }^{6}$ In cell culture, neurons were fixed for 15 min in $4 \%$ paraformaldehyde. Human sural nerve biopsies were obtained from four subjects with moderately severe diabetic neuropathy or from four subjects of similar age after traumatic amputation (two) or with connective tissue disease and sensory symptoms (two) as previously described. ${ }^{9}$

To detect caspase-3 staining, DRG neurons or tissue sections $(10 \mu \mathrm{m})$ were stained with $0.1 \mu \mathrm{g} / \mathrm{mL} \mathrm{CM} 1$ antibody (kindly provided by A. Srinivasan, IDUN Pharm, La Jolla, CA) and $7.5 \mu \mathrm{g} / \mathrm{mL}$ rhodamine-conjugated goat anti-rabbit antibody (Vector, Burlingame, CA) for $1 \mathrm{~h}$ as previously described. ${ }^{6,10}$ The nuclear chromatin was counterstained with $1 \mu \mathrm{g} / \mathrm{mL}$ bisbenzamide in PBS. Neurons were considered to have active caspase- 3 if staining could be clearly localized to the neuronal or SC cytosol on sequential sections using both DIC and immunofluorescence. To determine if staining is specific for cleaved caspase- 3 , the antibody was preimmunoabsorbed with peptide in sections ${ }^{10}$ to indicate cleaved (active) caspase- 3 . 
TdT-mediated dUTP-biotin nick end labeling (TUNEL) staining was used to detect apoptotic neurons. For staining of apoptotic nuclei in cell cultures, cells are grown on ACLAR dishes and fixed in $4 \%$ paraformaldehyde. Samples are labeled with digoxigenin-dUTP and then stained with FITC-conjugated antidigoxigenin antibody as previously described. ${ }^{6}$ Transmission electron microscopy (TEM) of DRG neurons and SC was performed as previously described. 5,6

A caspase-3 fluorometric assay kit (Pharmingen, San Diego, CA) was used to determine caspase-3 activation in SH-SY5Y neurons and SC. The assay was conducted according to the manufacturer's instructions and in conjunction with an Epics Elite flow cell sorting (FACS-Coulter Cytometry, Hialeah, FL) using excitation at $380 \mathrm{~nm}$ and emission at $440 \mathrm{~nm} .{ }^{8}$ All experiments were conducted with the following controls: cell lysate alone, Ac-DEVD-AMC alone, nonapoptotic cells, and positive apoptotic controls. Caspase cleavage was gated in live cells with propidium iodide (PI) exclusion $(18 \mu \mathrm{g} / \mathrm{mL})$. The mean of the peak caspase- 3 level and the percent of neurons expressing cleaved caspase- 3 were measured. Changes in the Mt membrane potential $\left(\Delta \Psi_{\mathrm{M}}\right)$ in $\mathrm{SC}$ were determined by incubating with $5 \mu \mathrm{g} / \mathrm{mL}$ rhodamine 123 (Rh123) for $30 \mathrm{~min}$ at $37^{\circ} \mathrm{C}$ followed by FACS. Alternatively, DRGs were incubated in $2.5 \mathrm{mg} / \mathrm{mL} \mathrm{JC}-1$ for $30 \mathrm{~min}$ at $37^{\circ} \mathrm{C}$, and $590: 530$ ratios were measured using a fluorometer (Fluoroskan Ascent, Labsystems, Helsinki, Finland) and confirmed by confocal microscopy.

Adenovirus (Ad) constructs for uncoupling protein-1 (UCP1) and green fluorescent protein (GFP) were purified in the University of Iowa Viral Core. For DRG neurons, we obtained a $95-100 \%$ infection rate at $24 \mathrm{~h}$ with an MOI of 1000 . Adenoviral constructs showed no intrinsic toxicity to DRG neurons. NGF p75 receptor function blocking antibody (p75NTR FBA) was kindly provided by Louis Reichardt (University of California, San Francisco).

Immunoblotting for caspase-9 was performed in lysates of whole 12-month streptozotocin (STZ) diabetic rat nerves (kindly provided by Douglas Zochodne, University of Calgary) with caspase-9 Ab (Santa Cruz, CA) at 1:1000 for $2 \mathrm{~h}$. The secondary antibody was a horseradish peroxidase conjugate, applied for 1 hour, and visualized with enhanced chemiluminescence reagents (Amersham, MA). ${ }^{8}$

\section{RESULTS}

\section{Hyperglycemia Is Associated with Oxidative Stress in Diabetic Neuropathy Models}

In human neuronal (SH-SY5Y) cells exposed to high glucose, there is an increase in ROS generation that peaks at 2-3 h (FIG. 1) and then declines corresponding to increased cell death. ${ }^{11}$ Increased ROS generation is associated with Mt depolarization and enlargement. ${ }^{11}$ Swelling of Mt has been confirmed in STZ diabetic and acutely hyperglycemic rats, where the DRG neurons contain ballooned Mt with disrupted cristae. ${ }^{6}$ Although, at very high magnification, lysosomal integrity is maintained, frequent large vacuoles measuring from 0.25 to $1 \mu \mathrm{m}$ in diameter are observed evenly distributed throughout the cytoplasm. ${ }^{6}$ At high magnification, these vacuoles are seen to occur in Mt that are ballooned and have disruption of the inner 


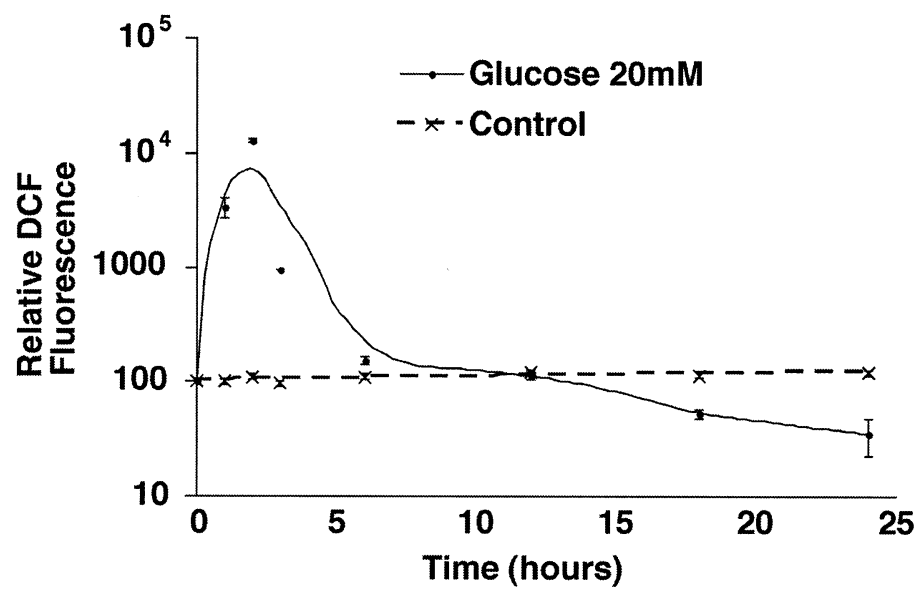

FIGURE 1. Glucose induces production of ROS in SH-SY5Y human neurons. SHSY5Y neurons were cultured up to $24 \mathrm{~h}$ in control serum-free media $\pm 20 \mathrm{mM}$ added glucose, treated with $2 \mu \mathrm{M} \mathrm{CM}-\mathrm{H}_{2}$ DCFDA, and analyzed with FACS to determine changes in oxidative stress. Experiments were standardized against control values at $0 \mathrm{~h}$. With addition of 20 $\mathrm{mM}$ added glucose, there was an initial threefold increase in the mean relative DCF fluorescence that peaked at $2 \mathrm{~h}(P<0.0001)$ and then declined, corresponding to increased neuronal death. Plotted values represent the mean relative DCF fluorescence as a percent of control at $0 \mathrm{~h}(100 \%) \pm$ SEM. Data were obtained from three separate experiments.

cristae structure. Although occasional vacuolization is observed in DRG cells from control animals, the Mt structure appears normal.

In human SC, there is also clear evidence of Mt enlargement and disruption of the normal cristae structure (FIG. 2). Whereas SC from nondiabetic subjects have normal Mt and rough endoplasmic reticulum, those from subjects with moderately severe diabetic neuropathy show formation of frequent vacuoles of variable size ranging up to $300 \mathrm{~nm}$ in diameter. While many of the vacuoles lack specific organellar structure, several enlarged Mt with still visible cristae structures are observed (FIG. 2C), consistent with increased oxidative damage to the Mt structure.

\section{Hyperglycemia Results in Mt Swelling and Dysregulation of the Inner Mt Membrane Potential}

Based on the findings of induction of oxidative stress and Mt damage in the peripheral nervous system (PNS), we next looked at the changes in the Mt membrane potential in neurons and SC. In DRG neurons exposed to increased concentrations of glucose, there is an initial hyperpolarization (more negative $\Delta \Psi_{\mathrm{M}}$ ) followed by depolarization (less negative $\Delta \Psi_{M}$ ), as seen in FiguRE 3A. DRG neurons were treated with $20 \mathrm{mM}$ added glucose for a period of $6 \mathrm{~h}$, and changes in the $\Delta \Psi_{\mathrm{M}}$ were measured using the potentiometric dye, JC-1. JC-1 exists as an aggregate at very negative membrane potentials $\left(\Delta \Psi_{\mathrm{M}}<-100 \mathrm{mV}\right)$ with a red fluorescence at $590 \mathrm{~nm}$, and as a monomer with green fluorescence at $530 \mathrm{~nm}$ with less negative potentials $\left(\Delta \Psi_{\mathrm{M}}>\right.$ 

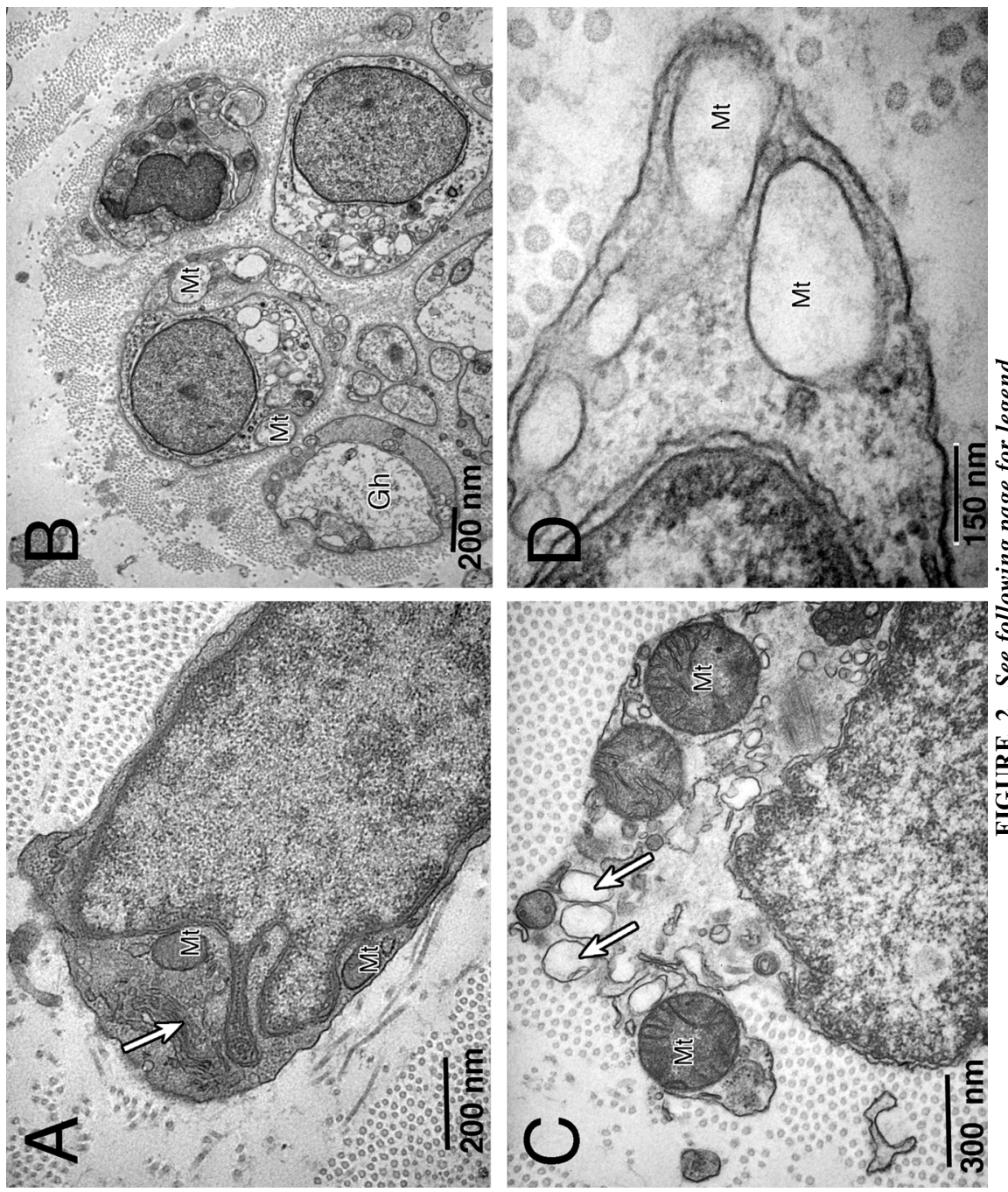
A

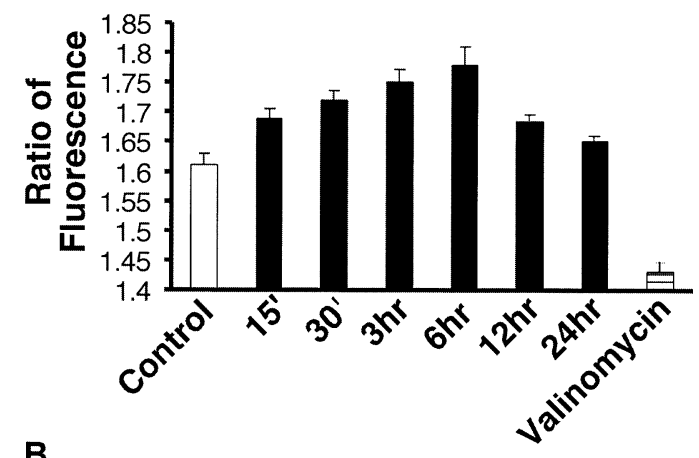

B

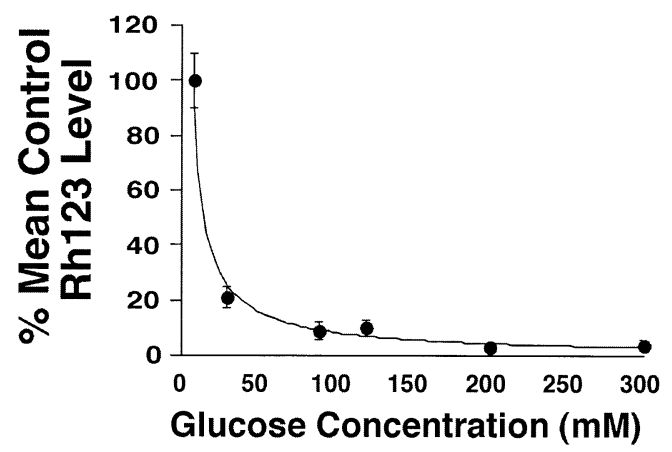

FIGURE 3. In neurons and SC, hyperglycemia is associated with disturbance of Mt polarization. (A) Dorsal root ganglion (DRG) neurons were incubated in JC-1 dye, and the $590 \mathrm{~nm}$ (aggregate) to $530 \mathrm{~nm}$ (monomer) ratio was measured to determine the $\Delta \Psi_{\mathrm{M}}$ under control and $20 \mathrm{mM}$ added glucose conditions at time points of $15 \mathrm{~min}$ to $12 \mathrm{~h}$. There is initial hyperpolarization on exposure to glucose followed by depolarization starting between 3 and 6 h. Complete depolarization is observed with valinomycin or oligomycin controls. (B) SC were cultured for $24 \mathrm{~h}$ with increasing glucose, followed by FACS analysis with Rh123. The data were plotted as a percent of the corresponding control value at the same time point. Even with $20 \mathrm{mM}$ added glucose, the $\Delta \Psi_{\mathrm{M}}$ was significantly reduced compared to control after $24 \mathrm{~h}(P<0.0001)$.

$-100 \mathrm{mV}$ ). Equal numbers of neurons were used at each time point and the ratio was standardized against protein levels. There was an increase in the 590:530 ratio with glucose exposure from $15 \mathrm{~min}$ to $3-6 \mathrm{~h}$, followed by a decline in the ratio consistent with depolarization, which persists to at least $24 \mathrm{~h}$. With valinomycin or oligomycin

FIGURE 2. In human SC, there is swelling and disruption of the Mt and of the rough endoplasmic reticulum (RER). SC TEMs were from normal human controls and patients with moderately severe diabetic neuropathy. (A) Control SC: normal Mt and RER (white arrow). (B, C, D) Diabetic SC: There are multiple enlarged Mt within the SC with disruption of the normal cristae structure and formation of cytoplasmic vacuoles (white arrows). 
(not shown), complete depolarization was observed. Similar results are obtained using Rh123 and TMRM, although evidence of depolarization is usually observed by $6 \mathrm{~h}$ using these cell-permeant dyes (data not shown). TMRM has the advantage of reducing fluorescent quenching sometimes observed with JC-1. In SC exposed to high concentrations of glucose, there is a dose-dependent decline in the $\Delta \Psi_{\mathrm{M}}$ consistent with MMD. With $20 \mathrm{mM}$ added glucose, the mean Rh123 measured using FACS decreased to $20 \%$ of control $(P<0.001)$, with almost complete loss of the $\Delta \Psi_{\mathrm{M}}$ with $300 \mathrm{mM}$ glucose (FIG. 3B).

\section{Hyperglycemia Is Associated with PCD in Diabetic Neuropathy Models and Human Disease}

Recent observations from our own and other laboratories indicate that apoptosis is one of the underlying causes of diabetic neuropathy. ${ }^{6,8,12-14}$ Clear pathological changes of apoptosis occur in DRG neurons and SC in STZ-treated diabetic rats. ${ }^{6,8}$ Hyperglycemia induces caspase- 3 cleavage in DRG cultures and a significant number of neurons are TUNEL-positive in STZ-treated rats after 1 month of hyperglycemia, indicating extensive and regular DNA fragmentation associated with apoptosis. The percent of apoptotic neurons in diabetic animals closely correlates with the serum glucose levels $\left(R^{2}>0.9\right)$, indicating that hyperglycemia is a likely trigger for apoptosis. In addition to the Mt dysfunction and apoptosis, our previously published data demonstrate a loss of neurons in rats after 3 months of diabetes. Using counting corrections for split nuclei, we estimated that the DRG number was $164 \pm 16$ in STZ diabetic animals, and $128 \pm 19$ in control animals, with a reduction in mean DRG area from $1110.3 \pm 81.2$ in control animals to $906.8 \pm 51.3 \mu \mathrm{m}^{2}$ in diabetics. $^{6}$

In tissue from C57BL/6 mice made acutely hyperglycemic with slow peritoneal infusion of $50 \%$ dextrose (mean serum glucose $=311.2 \pm 13.4 \mathrm{mg} / \mathrm{dL}, n=5$ ) and control animals (mean serum glucose $=161.8 \pm 7.3 \mathrm{mg} / \mathrm{dL}, n=5$ ), there is an increase in caspase-3 staining in DRG and, to a lesser extent, in SC (FIG. 4). Animals were sacrificed $24 \mathrm{~h}$ after attaining maximal hyperglycemia. Cytosolic caspase- 3 cleavage was coupled with evidence of nuclear chromatin condensation, although this was a less sensitive measure of neuronal injury. In addition to cleavage of caspase- 3 in both neurons and $\mathrm{SC},{ }^{8}$ there is also evidence of caspase- 9 cleavage in nerves from 12-month STZ diabetic rats. Although minimal cleavage of caspases is observed in most tissue that is removed from live animals and processed, there was a $40 \%$ increase in caspase- 9 cleavage in sural nerve from the diabetic animals (FIG. 4E).

Changes in animal models of diabetes are reproduced in human SC (FIG. 5). Compared to normal human SC with diffuse chromatin staining, there is increased chromatin condensation (FIG. 5B), with shrinkage of the SC cytosol. Eventually, there is loss of the $\mathrm{SC}$ nucleus and cytosol with preservation of the plasma membrane and supporting collagen, forming ghost cells. These changes are consistent with single cell deletion observed in apoptosis, but rarely in necrosis. No inflammatory infiltrates were observed in the affected nerves, such as might be seen with necrosis of the peripheral nerve. 


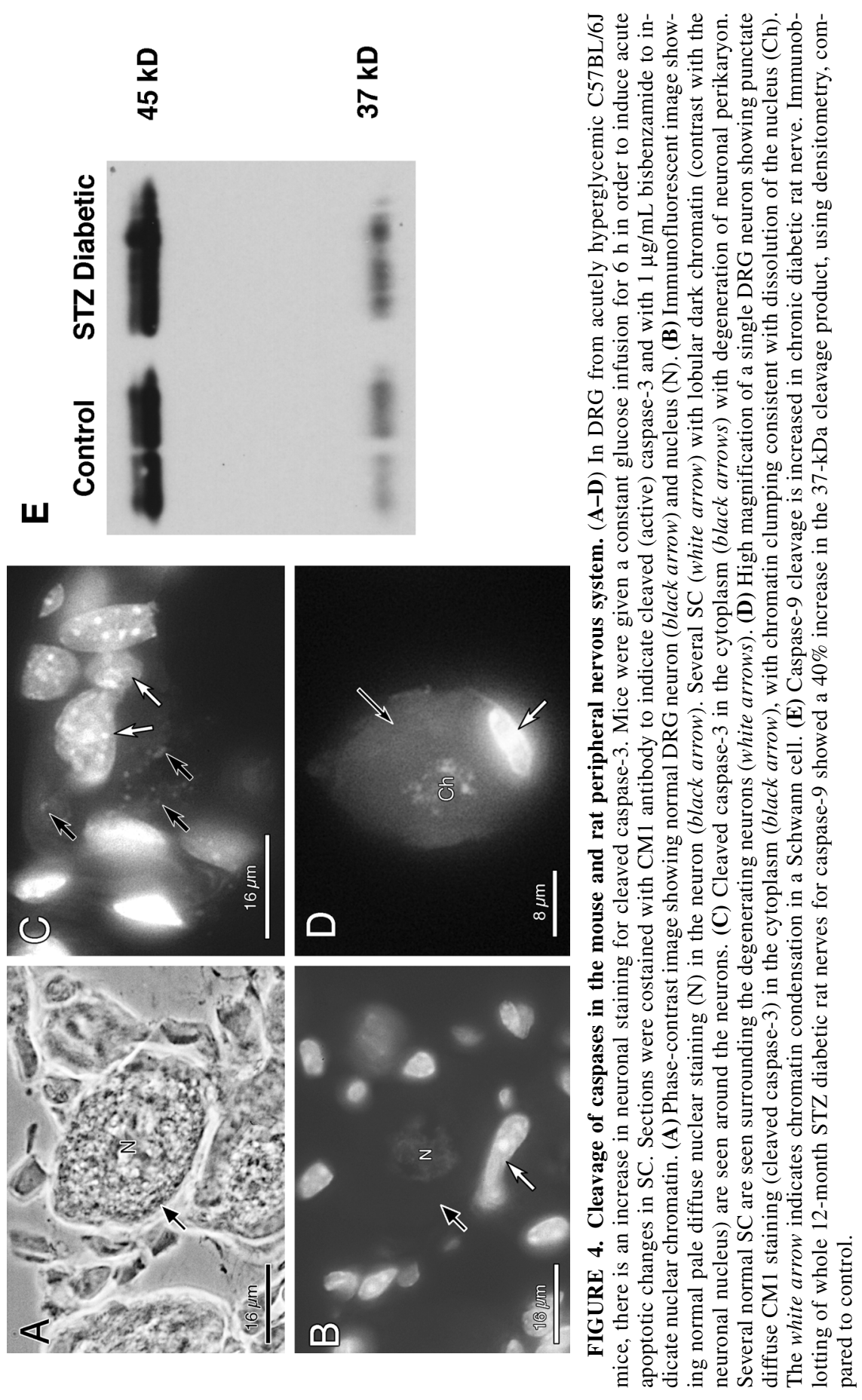



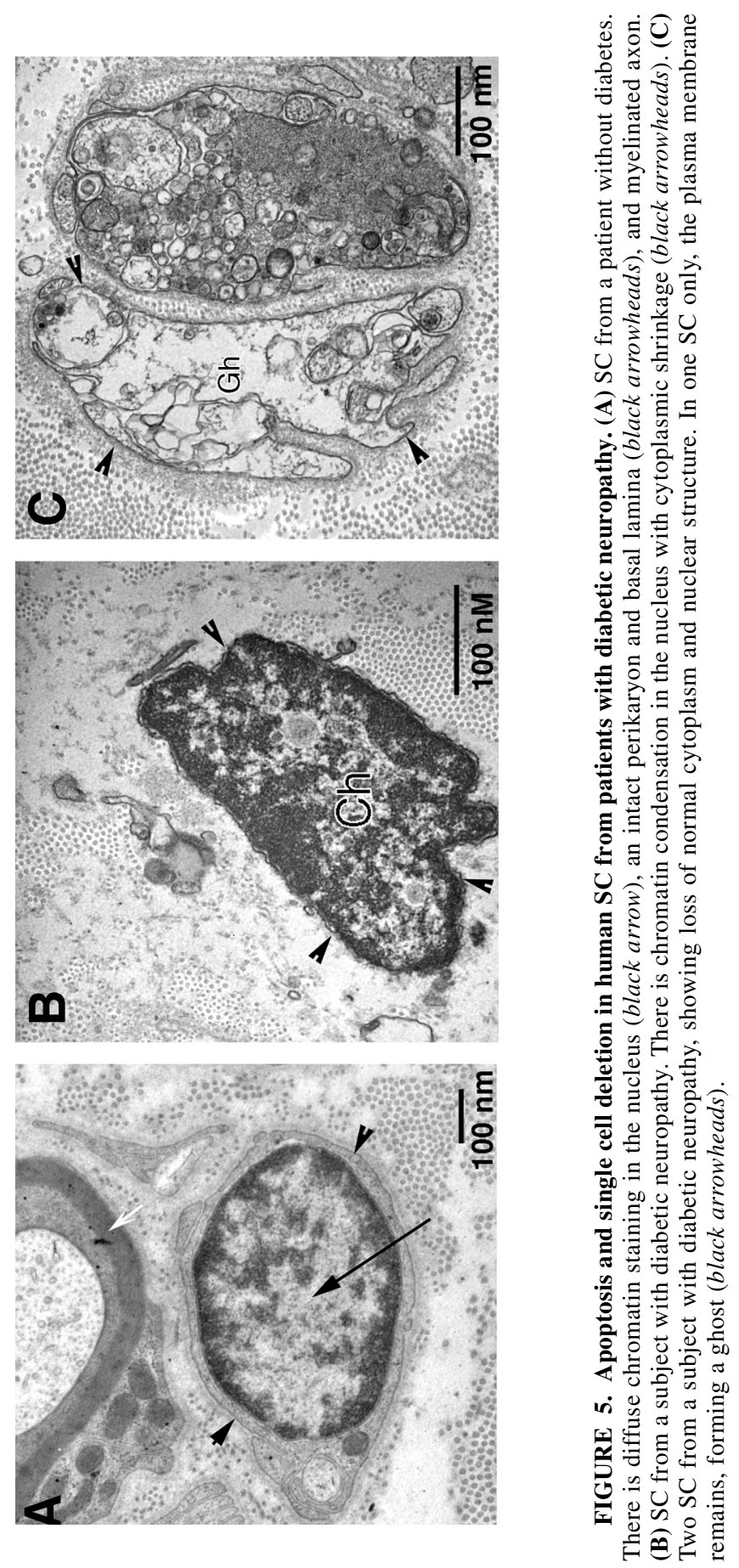


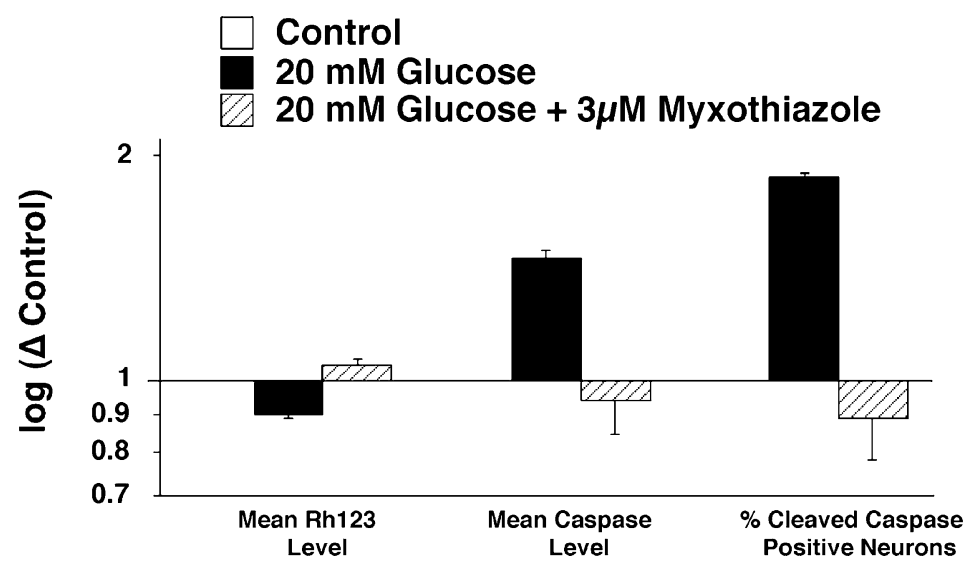

FIGURE 6. Myxothiazole, an inhibitor of the Mt respiratory chain, reduces MMD and cleavage of caspase-3 in SH-SY5Y neurons exposed to high glucose. The graph indicates the change from control as 1.0 on a log scale. SH-SY5Y neurons were cultured for 6 $\mathrm{h}$ in $20 \mathrm{mM}$ added glucose, and the $\Delta \Psi_{\mathrm{M}}$ was measured using Rh123 and FACS. There is a decrease in the $\Delta \Psi_{\mathrm{M}}$ at $6 \mathrm{~h}$ with $20 \mathrm{mM}$ added glucose, which is blocked by addition of $3 \mu \mathrm{M}$ myxothiazole. The mean level of cleaved caspase-3 and the percent of caspase-3-positive neurons are increased in the presence of $20 \mathrm{mM}$ added glucose and reduced by myxothiazole.

\section{Regulation of Glucose-induced Oxidative Stress and Mt Membrane Potential Inhibits PCD}

Glucose oxidation in the Mt increases the supply of electrons to the electron transfer chain, leading to increased proton pumping from the Mt and resulting in initial hyperpolarization of the $\Delta \Psi_{\mathrm{M}}$. Ultimately, increased electron loss from the electron transfer chain induces formation of superoxide anions and other ROS. This can be blocked by treatment with electron transfer inhibitors such as myxothiazole. In neurons cultured under control conditions, a reduction in electron transfer along the chain by myxothiazole would lead to a reduction in the $\Delta \Psi_{\mathrm{M}}$. Myxothiazole inhibits the Mt respiratory chain at cytochrome $\mathrm{b}-\mathrm{c}_{1} \cdot{ }^{15}$ In control neurons, addition of $3 \mu \mathrm{M}$ myxothiazole results in mild depolarization, whereas it maintains the $\Delta \Psi_{\mathrm{M}}$, which is usually decreased, in the presence of high glucose (Fig. 6). In Mt treated with high glucose that are biochemically compromised, oligomycin induces rapid depolarization consistent with Mt dependence on ATP synthase (data not shown). In high glucose states, myxothiazole is able to regulate generation of superoxides and prevent MMD. Coupled with this, myxothiazole blocks an increase in the mean level of caspase-3 cleavage and in the percentage of caspase-3-positive neurons (FIG. 6).

A further approach to regulating the $\Delta \Psi_{\mathrm{M}}$ in the presence of high glucose is with growth factors. Growth factors such as NGF may also serve as antioxidants and this function may contribute to their role as possible therapeutic entities in diabetic neuropathy. ${ }^{16-18}$ At $24 \mathrm{~h}$, exposure to increased glucose results in MMD that is blocked by a physiological concentration of NGF (FIG. 7). By contrast, pretreatment with $50 \mu \mathrm{g} / \mathrm{mL}$ p75NTR FBA blocks the effect of NGF on the $\Delta \Psi_{\mathrm{M}}$. These results 


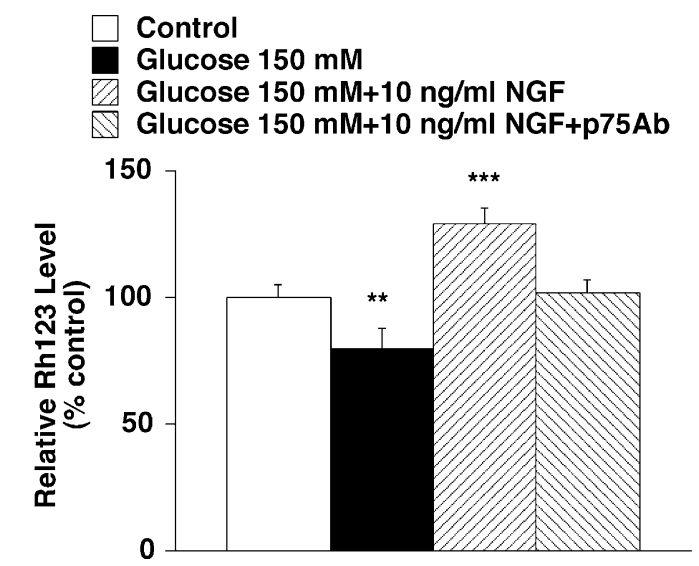

FIGURE 7. NGF prevents MMD in SC exposed to high glucose using FACS. The mean Rh123 level is decreased at $24 \mathrm{~h}$, in the presence of high glucose, but maintained by addition of a physiological concentration of NGF (10 ng/mL). NGF inhibition of MMD is blocked by $50 \mu \mathrm{g} / \mathrm{mL}$ p75NTR FBA.

indicate that NGF mediates some of its Mt stabilizing effects through the p75NTR. High glucose induces dose-dependent cleavage of caspases in SC (FIG. 8). SC were cultured for $6 \mathrm{~h}$ in increasing glucose, and caspase-3 cleavage was measured. Caspase- 3 cleavage was increased by $6 \mathrm{~h}$ with $20-50 \mathrm{mM}$ glucose and blocked by $10 \mathrm{ng} / \mathrm{mL}$ NGF or the caspase-3 inhibitor, DEVD-CHO. A similar inhibition of apoptosis, measured with TUNEL, was seen with $10 \mathrm{ng} / \mathrm{mL}$ NGF, but was reversed by pretreatment with p75NTR FBA (FIG. 8B). These results show that NGF inhibits $\mathrm{SC}$ apoptosis through the p75NTR.

The UCPs are inner Mt membrane proteins that can dissipate the protonelectrochemical gradient; that is, they uncouple Mt electron transfer from oxidative phosphorylation. ${ }^{19}$ UCPs share considerable amino acid sequence homology to one another, suggesting that they all possess an uncoupling function. However, the UCPs also have frequent homology to Mt transporters, as well as displaying typical Mt energy transfer protein signatures, suggesting that they may be Mt carriers. ${ }^{20,21}$ This function will help regulate the $\Delta \Psi_{\mathrm{M}}$ with hyperglycemic Mt injury and will prevent initial hyperpolarization. UCP1 is a classical UCP that is not normally expressed in neurons. In the presence of $20 \mathrm{mM}$ added glucose, there is an increase in cleavage of caspase-3 and TUNEL-positive DRG neurons (FIG. 9). Cleavage of caspases is blocked at $6 \mathrm{~h}$ by overexpression of UCP1 (FIG. 9), and induction of apoptosis is blocked at $24 \mathrm{~h}(P<0.001)$.

\section{DISCUSSION}

Recent evidence suggests that oxidative stress is responsible for the development and progression of neuropathy. ${ }^{22}$ Blocking oxidative stress in the diabetic animal prevents the development of neuropathy ${ }^{22}$ and restores sciatic and saphenous nerve 
A
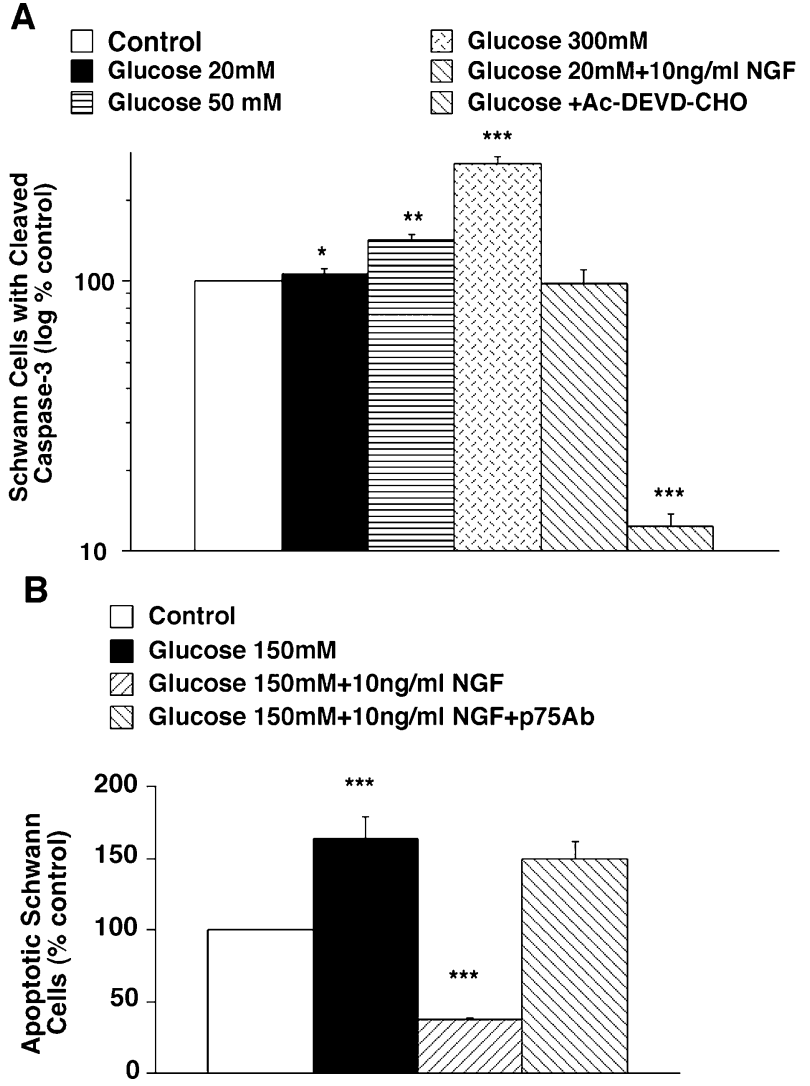

FIGURE 8. NGF inhibition of glucose-induced apoptosis is blocked by p75NTR FBA. (A) Cleavage of caspase- 3 is increased in SC exposed to high glucose in a dose-dependent fashion. The percent of SC with cleavage of caspase- 3 was determined at $6 \mathrm{~h}$ using increasing concentrations of glucose $\pm 10 \mathrm{ng} / \mathrm{mL}$ NGF or DEVD-CHO, and is expressed as a $\log$ scale. (B) Apoptosis in SC is inhibited by addition of NGF. SC apoptosis was determined at $24 \mathrm{~h}$ using PI and FACS analysis. Treatment with $50 \mu \mathrm{g} / \mathrm{mL}$ p 75 NTR FBA blocked NGF neuroprotection. $* P<0.05, * * P<0.01, * * * P<0.001$ compared to control.

conduction velocities in STZ diabetic rats. ${ }^{23,24}$ Our data indicate that increased concentrations of glucose rapidly induce production of ROS. This can be modulated by reducing electron flux along the electron transfer chain and generation of superoxide anions using myxothiazole. In turn, myxothiazole is able to reduce MMD seen with high glucose and to block induction of caspase cleavage. This most likely results from the ability of myxothiazole to reduce generation of ROS and to stabilize the $\Delta \Psi_{\mathrm{M}}$.

In DRG and SC exposed to high glucose, both in vivo and in vitro, there is $\mathrm{Mt}$ swelling and ultimately a decrease in the $\Delta \Psi_{\mathrm{M}}$, an event that in other paradigms initiates PCD. ${ }^{1}$ It has previously been shown that release of cytochrome $\mathrm{c}$ into the 


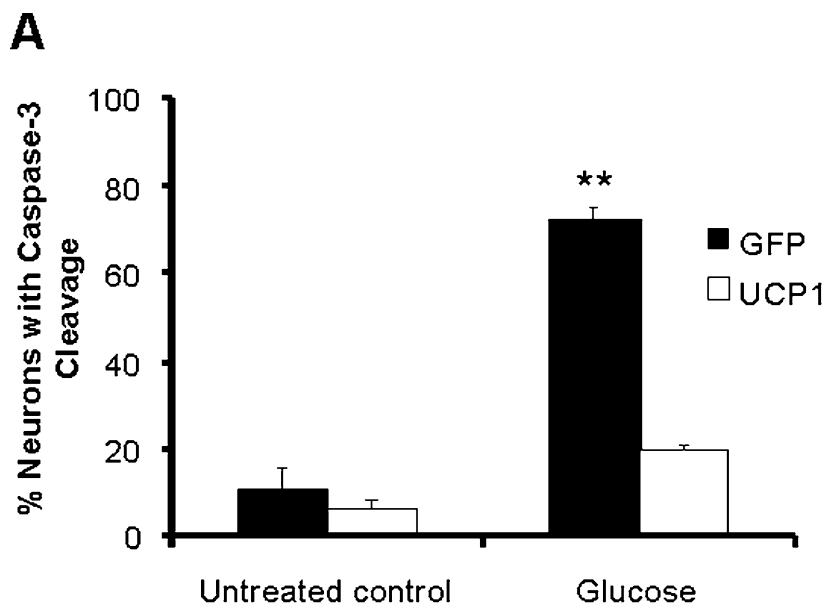

B

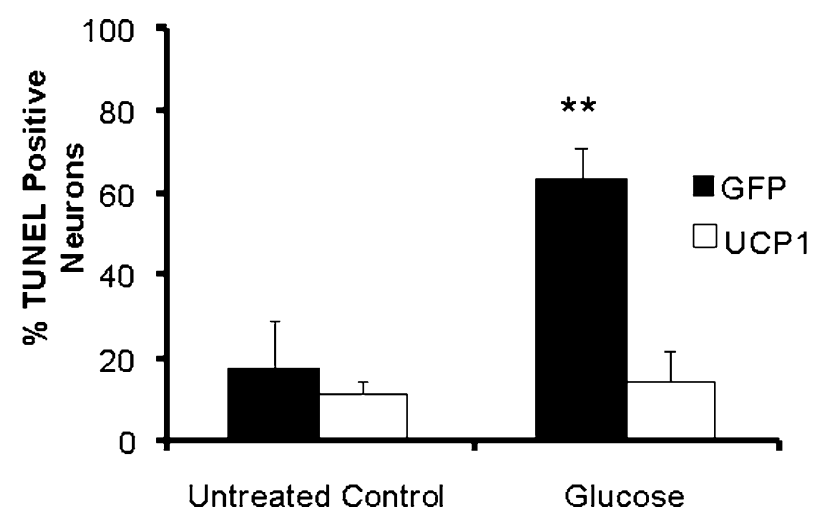

FIGURE 9. Adenovirus-mediated UCP1 expression prevents glucose-induced neuronal PCD. DRG neurons were infected with adenoviral constructs coding for either green fluorescent protein (GFP) as control or UCP1. (A) Caspase- 3 cleavage was measured at $6 \mathrm{~h}$ and was increased with $20 \mathrm{mM}$ added glucose and reduced by infection with UCP1. (B) Neurons were fixed $24 \mathrm{~h}$ following exposure to control media or $20 \mathrm{mM}$ added glucose. The fragmentation of genomic DNA was then assessed by TUNEL staining. $* * P<0.01$ compared to control.

cytosol is associated with formation of a cytochrome c/caspase-9/Apaf-1 complex (the apoptosome) and cleavage of downstream effector caspases such as caspase-3 and caspase-7. ${ }^{1}$ In human and rat neurons, hyperglycemia induces Mt dysfunction and apoptosis, ${ }^{6,14}$ and similar changes are observed in another important cell in the PNS - namely, the SC. In nerve from 12-month diabetic rats, there is an increase in caspase- 9 cleavage, and caspase- 3 cleavage is seen in cultured SC exposed to high 
glucose. Similar to changes in rat neurons, human SC show evidence of both Mt swelling and apoptosis.

Impaired trophic support, such as reduced NGF levels seen in diabetic neuropathy, results in increased peroxinitrite formation and cleavage of caspases. ${ }^{17}$ By contrast, we show that administration of NGF regulates MMD and blocks PCD. NGF may prevent PCD by blocking cell death signaling through the $75 \mathrm{NTR}^{25}$ and by upregulating PCD threshold proteins such as Bcl-xL and Bcl-2. ${ }^{17,26}$ While chronic diabetes in humans may be associated with down-regulation of UCPs, up-regulation of UCP1 is clearly mediated through increased expression of p85 $\alpha$-phosphatidylinositol 3-kinase (PI-3K) or mitogen-activated protein kinase (MAPK)/MAPextracellular signal-regulated kinase (MEK). These signal transduction molecules are critical intermediates of several growth factors including NGF and insulin-like growth factor (IGF-I). ${ }^{27,28}$ Growth factor regulation of the $\Delta \Psi_{\mathrm{M}}$ by UCPs, especially UCP2 and UCP3, expressed in DRG neurons, ${ }^{29}$ would reduce generation of ROS and prevent induction of PCD, and ultimately may ameliorate diabetic neuropathy.

\section{CONCLUSIONS}

These observations in the PNS have led to our proposing the following model for regulation of glucose-induced oxidative stress and PCD in neuropathy. Glucose induction of ROS is critical to the pathogenesis of diabetic neuropathy. Loss of electrons from the Mt electron transfer chain, coupled with initial hyperpolarization of the $\Delta \Psi_{\mathrm{M}}$, results in generation of excess ROS in the Mt. In turn, there is increased Mt injury, MMD, and swelling with release of apoptosis-inducing factors from the Mt into the cytosol, leading to formation of an apoptosome. Growth factors upregulate UCPs in the Mt and Bcl PCD threshold proteins like Bcl-2/Bcl-xL, stabilize the $\Delta \Psi_{\mathrm{M}}$, and block formation of ROS and induction of PCD. Further understanding of these mechanisms will be useful in developing potential therapeutic strategies in diabetic neuropathy.

\section{ACKNOWLEDGMENTS}

This work was supported by grants from the NIH (Nos. NS 01938, NS 42056, and NIDDK 5P60DK-20572), The Juvenile Diabetes Research Foundation Center for the Study of Complications in Diabetes, VA Merit Review, funds from the Ann Arbor Geriatric Research and Clinical Center (GRECC), and the Program for Understanding Neurological Diseases (PFUND).

We also would like to thank Judy Boldt for secretarial support, and Eva Feldman, Mila Blaivas, Kelli Sullivan, and Catherine Delaney at the University of Michigan for their helpful discussions and assistance with this work; Douglas Zochodne at the University of Calgary for providing tissue from 12-month diabetic rats; the Michigan Imaging Analysis Laboratory (MIL) for assistance with electron microscopy; and the University of Michigan Core Flow Cytometry for assistance with FACS (supported in part by the UM-Comprehensive Cancer Center [NIH CA46592], the UM-Multipurpose Arthritic Center [NIH AR20557], and the UM-BRCF Core Flow Cytometry Facility). 


\section{REFERENCES}

1. Green, D.R. \& J.C. ReED. 1998. Mitochondria and apoptosis. Science 281: 1309-1312.

2. Feldman, E.L., M.J. Stevens \& D.A. Greene. 1997. Pathogenesis of diabetic neuropathy. Clin. Neurosci. 4: 365-370.

3. Greene, D.A., I. Obrosova, M.J. Stevens \& E.L. Feldman. 2000. Pathways of glucose-mediated oxidative stress in diabetic neuropathy. In Antioxidants in Diabetes Management, pp. 111-119. Dekker. New York.

4. Fujimura, M., Y. Morita-Fujimura, M. Kawase, et al. 1999. Manganese superoxide dismutase mediates the early release of mitochondrial cytochrome $\mathrm{c}$ and subsequent DNA fragmentation after permanent focal cerebral ischemia in mice. J. Neurosci. 19: 3414-3422.

5. Russell, J.W., H-L. Cheng \& D. Golovoy. 2000. Insulin-like growth factor-I promotes myelination of peripheral sensory axons. J. Neuropathol. Exp. Neurol. 59: $575-584$.

6. Russell, J.W., K.A. Sullivan, A.J. Windebank, et al. 1999. Neurons undergo apoptosis in animal and cell culture models of diabetes. Neurobiol. Dis. 6: 347-363.

7. van Golen, C.M. \& E.L. Feldman. 2000. Insulin-like growth factor I is the key growth factor in serum that protects neuroblastoma cells from hyperosmotic-induced apoptosis. J. Cell. Physiol. 182: 24-32.

8. Delaney, C.L., J.W. Russell, H-L. Cheng \& E.L. Feldman. 2001. Insulin-like growth factor-I and over-expression of Bcl-xL prevent glucose-mediated apoptosis in Schwann cells. J. Neuropathol. Exp. Neurol. 60: 147-160.

9. Russell, J., J. Karnes \& P. DyCK. 1996. Sural nerve myelinated fiber density differences associated with meaningful changes in clinical and electrophysiological measurements. J. Neurol. Sci. 135: 114-117.

10. Srinivasan, A., K.A. Roth, R.O. SAYers, et al. 1998. In situ immunodetection of activated caspase-3 in apoptotic neurons in the developing nervous system. Cell Death Differ. 5: 1004-1016.

11. Feldman, E.L., J.W. Russell, K.A. Sullivan \& D. Golovoy. 2000. New insights into the pathogenesis of diabetic neuropathy. Curr. Opin. Neurol. 12: 553-563.

12. Srinivasan, A., M.J. Stevens \& J.W. Wiley. 2000. Diabetic peripheral neuropathy: evidence for apoptosis and associated mitochondrial dysfunction. Diabetes 49: 1932-1938.

13. Russell, J.W., C. Delaney, A.R. Berent, et al. 2001. Diabetes and impaired glucose tolerance induce axonal neuropathy and programmed cell death (PCD) in the obese Zucker rat. Endocr. Soc. Abstr. P1-363: 226.

14. Russell, J.W. \& E.L. Feldman. 1999. Insulin-like growth factor-I prevents apoptosis in sympathetic neurons exposed to high glucose. Horm. Metab. Res. 31: 90-96.

15. Vanden Hoek, T.L., L.B. Becker, Z. ShaO, et al. 1998. Reactive oxygen species released from mitochondria during brief hypoxia induce preconditioning in cardiomyocytes. J. Biol. Chem. 273: 18092-18098.

16. Pan, Z., D. SAmpath, G. JaCkson, et al. 1997. Nerve growth factor and oxidative stress in the nervous system. Adv. Exp. Med. Biol. 429: 173-193.

17. PARK, D.S., E.J. Morris, L. Stefanis, et al. 1998. Multiple pathways of neuronal death induced by DNA-damaging agents, NGF deprivation, and oxidative stress. J. Neurosci. 18: 830-840.

18. Lieberthal, W., V. Triaca, J.S. KoH, et al. 1998. Role of superoxide in apoptosis induced by growth factor withdrawal. Am. J. Physiol. 275(5, pt. 2): F691-F702.

19. Nishikawa, T., D. Edelstein, X.L. Du, et al. 2000. Normalizing mitochondrial superoxide production blocks three pathways of hyperglycaemic damage. Nature 404: 787-790.

20. Boss, O., S. Samec, A. Paoloni-Giacobino, et al. 1997. Uncoupling protein-3: a new member of the mitochondrial carrier family with tissue-specific expression. FEBS Lett. 408: $39-42$.

21. BAIROCH, A. 1993. The PROSITE dictionary of sites and patterns in proteins: its current status. Nucleic Acids Res. 21: 3097-3103. 
22. Stevens, M.J., I. Obrosova, X. CaO, et al. 2000. Effects of DL-alpha-lipoic acid on peripheral nerve conduction, blood flow, energy metabolism, and oxidative stress in experimental diabetic neuropathy. Diabetes 49: 1006-1015.

23. Low, P.A., K.K. NickANDER \& H.J. TRITSCHLER. 1997. The roles of oxidative stress and antioxidant treatment in experimental diabetic neuropathy. Diabetes 46(suppl. 2): S38-S42.

24. CAmeron, N.E. \& M.A. CotTer. 1997. Metabolic and vascular factors in the pathogenesis of diabetic neuropathy. Diabetes 46(suppl. 2): S31-S37.

25. BREDESEN, D.E. \& S. RabizadeH. 1997. p75NTR and apoptosis: Trk-dependent and Trk-independent effects. Trends Neurosci. 20: 287-290.

26. Muller, Y., K. TANGRe \& J. Clos. 1997. Autocrine regulation of apoptosis and bcl-2 expression by nerve growth factor in early differentiating cerebellar granule neurons involves low affinity neurotrophin receptor. Neurochem. Int. 31: 177-191.

27. Valverde, A.M., M. Lorenzo, P. Navarro \& M. Benito. 1997. Phosphatidylinositol 3-kinase is a requirement for insulin-like growth factor I-induced differentiation, but not for mitogenesis, in fetal brown adipocytes. Mol. Endocrinol. 11: 595-607.

28. Teruel, T., A.M. Valverde, P. Navarro, et al. 1998. Inhibition of PI 3-kinase and RAS blocks IGF-I and insulin-induced uncoupling protein 1 gene expression in brown adipocytes. J. Cell. Physiol. 176: $99-109$.

29. Vincent, A.M., C. Gong, M. Brownlee \& J.W. Russell. 2001. Glucose induced neuronal programmed cell death is regulated by manganese superoxide dismutase and uncoupling protein-1. Endocr. Soc. Abstr. P1-289: 210. 